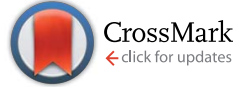

Cite this: Chem. Sci., 2015, 6, 3769

\title{
Micro-competition system for Raman quantification of multiple glycans on intact cell surface $\uparrow$
}

\author{
Yunlong Chen,,$_{+}^{a}$ Lin Ding, $t^{a}$ Junqiang $\mathrm{Xu},{ }^{a}$ Wanyao Song, ${ }^{a}$ Min Yang, Junjie $\mathrm{Hu}^{\mathrm{a}}$ \\ and Huangxian $\mathrm{Ju}^{\star a}$
}

A micro-competition system is designed for simultaneous quantification of multiple glycans on intact cell surfaces, by integrating two-surface-one-molecule competition with surface enhanced Raman scattering (SERS). The micro-competition is achieved among multiple-polysaccharide-coated gold nanostars functionalized silica bubbles, target cells and gold nanoprobes at a micron scale. The gold nanoprobes are prepared by coating distinct Raman molecules and lectins on gold nanoparticles for signal resolution and glycan recognition, respectively. The silica bubble surface serves as an artificial glycan surface and a SERS substrate. Upon the competitive recognition of lectin to the corresponding glycan, the gold nanoprobes can be specifically captured by the bubbles and cells in a homogeneous system, and the amounts of different gold nanoprobes on bubbles are simultaneously detected by SERS to reflect the corresponding glycan amounts on the cell surface. This micro-competition system with multiple quantification capability provides a powerful tool for investigation of the complex glycan-related biological processes.

Received 21st March 2015

Accepted 30th April 2015

DOI: $10.1039 / c 5 s c 01031 d$

www.rsc.org/chemicalscience suffers from the undervaluation of certain glycans during the tedious cell lysis, enzymatic cleavage and derivatization processes. ${ }^{10}$ Alternatively, encoding-based lectin array techniques have been developed for multiplexed glycan profiling. ${ }^{11-13}$ However, those protein immobilization based methods suffer from the denaturation or inactivation of proteins, due to chemical modification and spatial inaccessibility on arrays. ${ }^{\mathbf{1 4 , 1 5}}$ To overcome these disadvantages, our previous work designed barcode-lectin probes for in situ fluorescence analysis of multiple cell surface glycans through a DNA microarray for decoding. ${ }^{16}$ In fact the preparation of barcode-lectin probes is relatively expensive and time consuming. Thus it is necessary to develop novel economic and practical coded probes for quantification of multiple glycans on intact cell surfaces.

Besides the fluorescence based technique, ${ }^{\mathbf{1 7}}$ surfaceenhanced Raman scattering (SERS) has also been used for evaluation of single cell surface glycans. ${ }^{18}$ This method cannot give quantitative results. In view of the advantages of SERS that can provide the complete vibrational information of molecules, ${ }^{\mathbf{1 9 , 2 0}}$ and Raman signal molecules (RSMs) which can be used to code for multiplexed detection, ${ }^{\mathbf{2 1 - 2 3}}$ we prepared a set of Raman barcoding probes to couple with a newly designed micro-competition system for fast, quantitative detection of multiple glycans on cell surfaces.

Competition is an ingenious quantification method, which can eliminate virtually any chance of false positive background 
signals. The micro-competition system consisted of three components: Raman signal molecule and lectin dual-coded gold nanoparticles (AuNPs), represented by nanoprobes, silica bubbles functionalized by multiple-polysaccharide-coated gold nanostars (MGAuNS@B), and cells. Nanoprobes were respectively coded with three kinds of RSMs with distinguishable characteristic peaks, and subsequently functionalized with different lectins to specifically recognize target glycans (Fig. 1a). The AuNSs are powerful SERS substrates owing to the strong plasmonic electromagnetic field due to their anisotropic nanostructure. ${ }^{24-27}$ The MGAuNS@B were prepared by in situ coating multiple polysaccharides on AuNS surfaces, and stepwise assembling the coated AuNSs onto silica bubbles by electrostatic adsorption. After mixing MGAuNS@B, nanoprobes and cells in PBS, the designed anisotropic multiple glycan surface could compete with cell surface glycans for binding different types of nanoprobes, via lectin-glycan recognition in a one-molecule-two-surface format at a micron scale, thus called a micro-competition system. Following completion of binding, MGAuNS@B could be quickly separated by buoyancy ${ }^{28-30}$ and the amount of bound nanoprobes could be detected by Raman spectrometry (Fig. 1b).

Compared with the complanate glycan surface previously designed for competitive recognition, ${ }^{31-34}$ the anisotropic surface of MGAuNS@B with dimensions close to the cells provided a better simulation of cell surface glycans for more efficient competitive recognition. ${ }^{35,36}$ Moreover, the

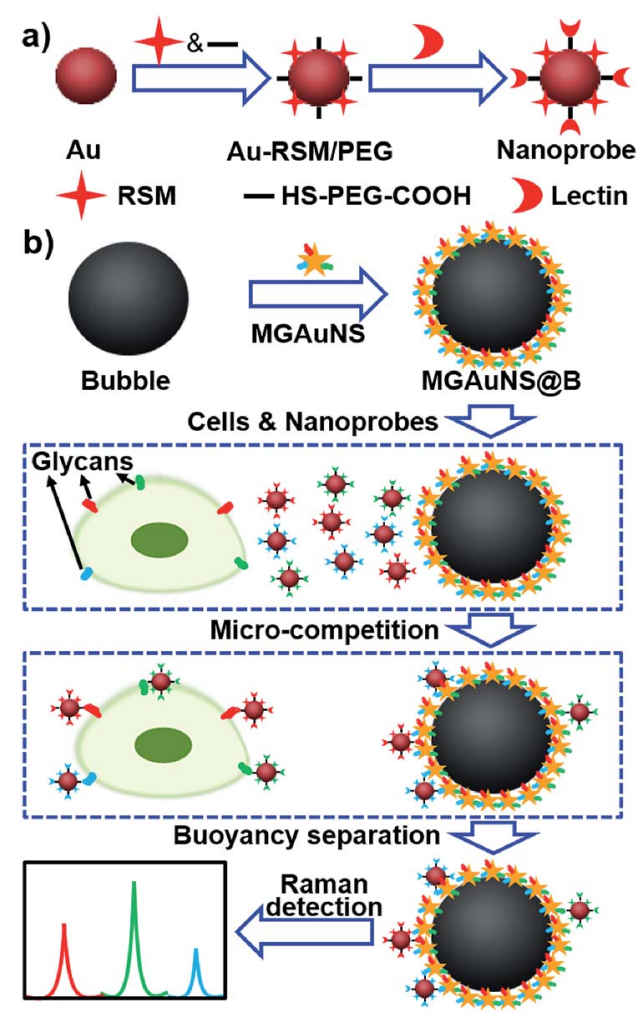

Fig. 1 Schematic illustration of (a) synthesis of nanoprobes and (b) micro-competition system for multiple Raman detection of cell surface glycans.
MGAuNS@B could act as SERS substrates for nanoprobes that could not generate Raman signals individually due to the small size. The amounts of glycans on cell surfaces could be accurately reflected by the amounts of nanoprobes selectively bound on the artificial glycan surfaces for sensitive Raman detection. The proposed glycan profiling method possesses whole-surface accessibility, rapid detection, enhanced sensitivity, high throughput and the advantage of cost effectiveness.

\section{Results and discussion}

\section{Characterization of nanoprobes and MGAuNS@B}

AuNPs were firstly modified with sulfhydryl contained polyethylene glycol (PEG) and subsequently coded with RSMs through Au-S binding and lectins via EDC-mediated carbodiimide chemistry. Three pairs of RSMs and lectins were involved: 2-naphthol (NT) and Lens culinaris agglutinin (LCA), 4-aminothiophenol (ATP) and Sambucus nigra agglutinin (SNA), and 5,5'-dithiobis(2-nitrobenzoic acid) (DTNB) and succinylated wheat germ agglutinin (SWGA). LCA and SNA can specifically recognize mannose (Man) and $N$-acetylneuraminic acid (Sia) respectively (Table $\mathrm{S} 1 \dagger$ ), while SWGA cannot bind to sialic acid residues but retains its specificity toward $\mathrm{N}$-acetylglucosamine (GlcNAc) (http://www.vectorlabs.com/catalog. aspx?catID=253). The UV spectra showed the characteristic peak of proteins at $280 \mathrm{~nm}$, indicating the efficient binding of lectins (Fig. S1†). Polysaccharide-coated AuNSs were synthesized by directly adding three kinds of polysaccharides to the growing solution of AuNSs. TEM images illustrate an obvious sugar layer on the AuNSs compared with AuNSs without polysaccharide coating (Fig. S2a and S2b $\dagger$ ). Zeta potential measurements showed a more negative charge and more narrow size distribution of AuNSs after polysaccharide coating (Fig. S2c †), which was possibly owing to the stability and protection of polysaccharides in the one-pot synthesis. The polysaccharide coating did not affect the SERS ability of AuNSs, as verified by the almost unchanged Raman signal intensity after sugar coating (Fig. S2d $\dagger$ ). Since the polysaccharide-coated AuNSs were negatively charged, they could adsorb to the positively charged poly(diallyldimethylammonium chloride) (PDDA)-coated silica bubbles. TEM images illustrate an obvious morphology change with the step-by-step assembly of MGAuNS@B (Fig. 2a-c). After incubation with the mixture of three kinds of nanoprobes, the MGAuNS@B surface displayed the bound nanoprobes (Fig. 2d).

To verify the feasibility of Raman coding, MGAuNS@B was incubated with either a single type of nanoprobe or a mixture of three types of nanoprobes. MGAuNS@B did not show any Raman response, while the nanoprobes showed the characteristic Raman peaks of the corresponding RSM (Fig. 3). The nanoprobe mixture also showed the characteristic peaks at $715.5 \mathrm{~cm}^{-1}, 1189.7 \mathrm{~cm}^{-1}$ and $1328.6 \mathrm{~cm}^{-1}$, indicating an easyto-observe distinction on the overlay spectrum. These peaks were chosen as the Raman barcode peaks for detection of three glycans on intact cell surfaces. 

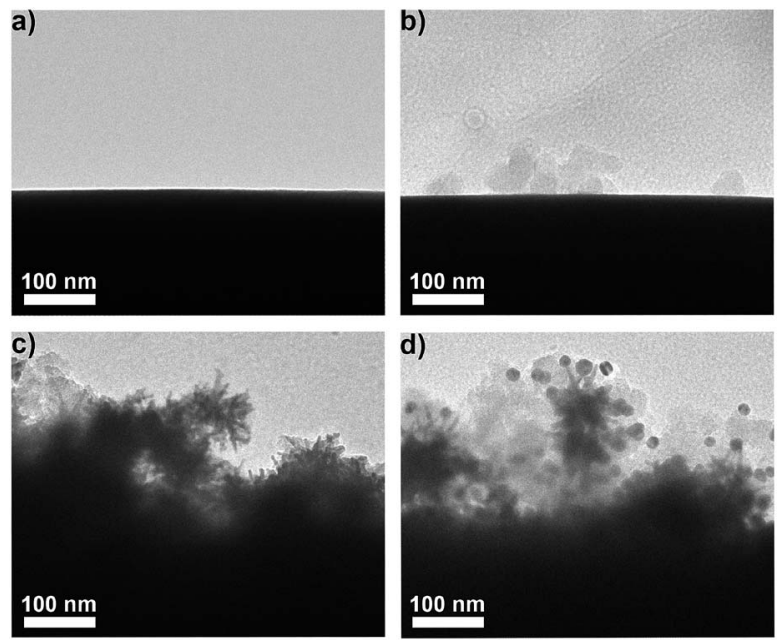

Fig. 2 TEM images of (a) bubble, (b) PDDA-bubble, (c) MGAuNS@B, and (d) nanoprobes-bound MGAuNS@B.

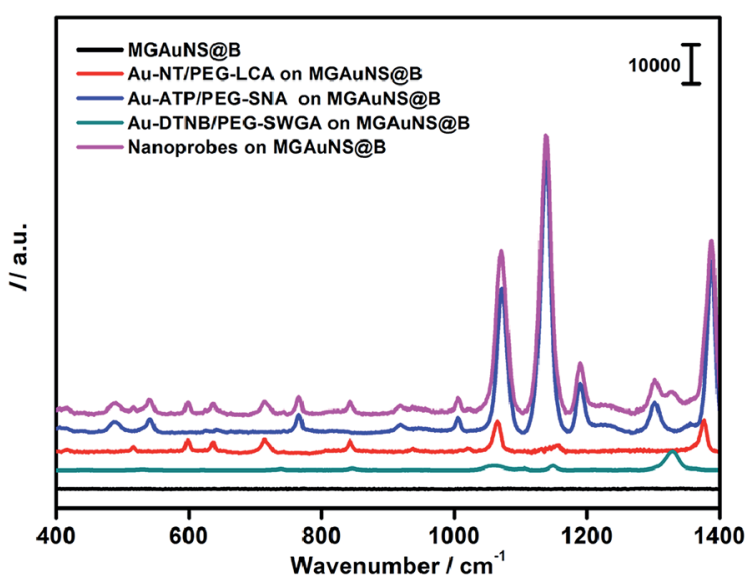

Fig. 3 Raman spectra of MGAUNS@B before and after incubation with three nanoprobes and their mixture.

\section{Recognition specificity}

The binding specificity of the nanoprobes to MGAuNS@B was verified with three types of silica bubbles, which were functionalized with different single polysaccharide-coated AuNSs. After incubation with the mixture of three nanoprobes, these bubbles could only bind the corresponding nanoprobe, while the bubbles modified with bare AuNSs did not showed any binding to the nanoprobe (Fig. S3†).

The recognition specificity was further validated by monosaccharide inhibition testing (Fig. S4 $\dagger$ ). After the mixture of nanoprobes was pre-inhibited with Man, Sia, or GlcNAc for 2 hours, it was incubated with MGAuNS@B. The Raman spectra of the resulting bubbles did not show the Raman barcode peak of the nanoprobe corresponding to the monosaccharide, while the Raman peaks coded with other lectins increased with the increasing concentration of the nanoprobes, indicating the specific inhibition of the nanoprobes by the corresponding monosaccharides with negligible recognition from the three recognition pairs.

\section{Quantitative detection of multiple cell surface glycans}

To achieve highly sensitive detection, the optimal time of binding between nanoprobes and MGAuNS@B was determined to be 60 minutes, at which the competitive binding of nanoprobes to cells and MGAuNS@B could be completed (Fig. S5†).

With the incubation time of 60 minutes, the standard binding curves of nanoprobes with MGAuNS@B are shown in Fig. 4a and b. For each kind of nanoprobe, the Raman intensity (I) of MGAuNS@B at the corresponding barcode peak was proportional to the nanoprobe concentration $\left(c_{\text {probe }}\right)$ in the range from 0.5 to $2.5 \mathrm{nM}$ (Fig. $4 \mathrm{~b}$ ):

$$
I=k_{1} c_{\text {probe }}+b_{1}
$$

In the presence of MCF-7 cells, the nanoprobes were competitively captured by the cells. The concentrations in the incubation mixture and MGAuNS@B were the total concentration $\left(c_{\text {probeo }}\right)-a_{\text {probe on each cell }} c_{\text {cell }}$, where $a_{\text {probe on each cell }}$ is the amount of nanoprobe bound on each cell. Thus

$$
I=k_{1}\left(c_{\text {probe } 0}-a_{\text {probe on each cell }} c_{\text {cell }}\right)+b_{1}
$$

i.e.

$$
I=-k_{1} a_{\text {probe on each cell }} c_{\text {cell }}+\left(b_{1}+k_{1} c_{\text {probe } 0}\right)
$$

At a constant $c_{\text {probeo }}$ of $1 \mathrm{nM}, I$ was inversely proportional to MCF-7 cell concentration $\left(c_{\text {cell }}\right)$ in the range from $1.0 \times 10^{3}$ to $9.0 \times 10^{3}$ cells per $\mathrm{mL}$ (Fig. $4 \mathrm{c}$ and $\mathrm{d}$ ):

$$
I=k_{2} c_{\text {cell }}+b_{2}
$$

From eqn (3) and (4):

$$
a_{\text {probe on each cell }}=-k_{2} / k_{1}
$$
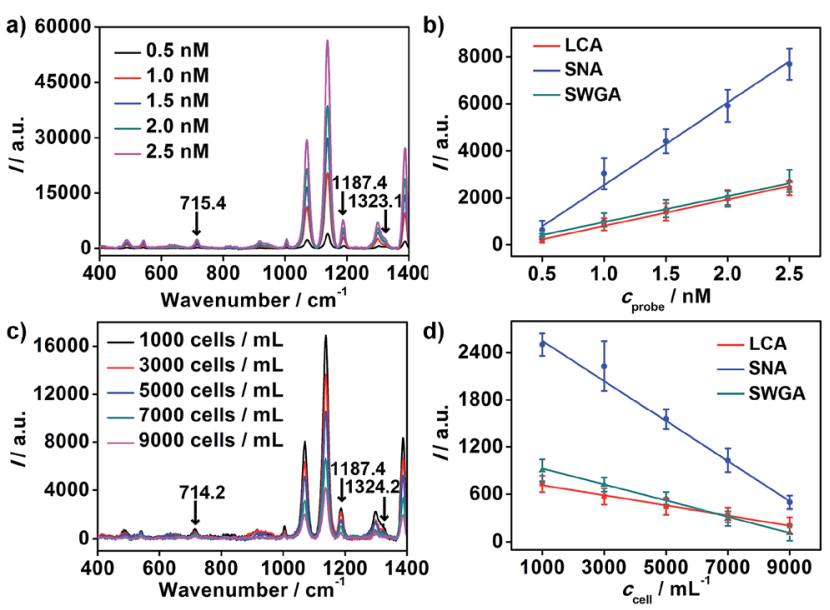

Fig. 4 (a) Raman spectra of MGAuNS@B after incubation with the nanoprobe mixture at different concentrations. (b) Plots of Raman intensity at $715.4 \mathrm{~cm}^{-1}, 1187.4 \mathrm{~cm}^{-1}$ and $1323.1 \mathrm{~cm}^{-1} v s$. nanoprobe concentration. (c) Raman spectra of MGAuNS@B after competition with different MCF-7 concentrations. (d) Plots of Raman intensity vs. cell concentration. 
Hence, the amount of different nanoprobes bound on each cell could be calculated, which could be regarded as the amounts of the corresponding glycan on each cell.

The slopes of the standard binding curves were determined as $1.1 \times 10^{3}, 3.5 \times 10^{3}$ and $1.1 \times 10^{3}$ for LCA, SNA and SWGA coded nanoprobes, and the slopes of MCF-7 cell competition curves were $-0.064,-0.26$ and -0.10 , respectively, for the corresponding nanoprobes. According to eqn (5), the average numbers of MCF-7 cell surface Man, Sia and GlcNAc could be calculated as $3.4 \times 10^{7}, 4.4 \times 10^{7}$ and $5.5 \times 10^{7}$ per cell. The relative expression extent was in good agreement with the results from flow cytometric analysis using fluorescein-labeled lectins (Fig. S6†), demonstrating the feasibility of the proposed method for simultaneous detection of multiple glycans on living cells.

The proposed strategy could also be used for cell quantification. The Raman characteristic peaks of three RSMs overlaid at $1070.8 \mathrm{~cm}^{-1}$ (Fig. 3), so the peak intensity at $1070.8 \mathrm{~cm}^{-1}$ could be used for quantification of cells. $I$ at $1070.8 \mathrm{~cm}^{-1}$ was found to be inversely proportional to logarithmic cell concentration in the range from $10^{2}$ to $10^{6}$ cells per mL (Fig. 5). Considering that the volume of cell suspension in the microcompetition system was $100 \mu \mathrm{L}$, the proposed method could realize a detection limit down to 10 cells.

\section{Monitoring of multiple cell surface glycans}

To further verify the application of the proposed strategy, cell surfaces glycans were regulated by treating the cells with glycan endonucleases. After MCF-7 cells were incubated with $100 \mathrm{U} \mathrm{mL}^{-1}$ mannosidase, neuraminidase, and $N$-acetyl-glucosaminidase, which can specifically cleave Man, Sia and GlcNAc, respectively, from cell surfaces at $37{ }^{\circ} \mathrm{C}$ for 30 minutes, they were subjected to the micro-competition system. For each kind of endonuclease, the change of the corresponding Raman barcode peak obtained on MGAuNS@B with the increasing $c_{\text {cell }}$ was very small (Fig. 6), which was negligible and lower than that before treatment (Fig. $4 \mathrm{c}$ and d), indicating the specific cleavage of the glycans by the corresponding endonuclease.

From eqn (5) and the slopes of competition curves with the three kinds of treated cells for three peaks corresponding to LCA, SNA and SWGA-coded nanoprobes, the amounts of Man, Sia and GlcNAc on the corresponding glycan endonuclease-
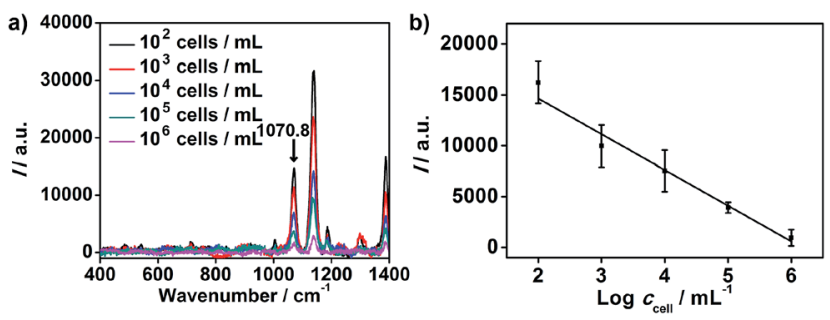

Fig. 5 (a) Raman spectra of MGAuNS@B after competition with MCF-7 cells of different concentrations. (b) Plot of Raman intensity at 1070.8 $\mathrm{cm}^{-1}$ shown in Fig. 3 vs. cell concentration.
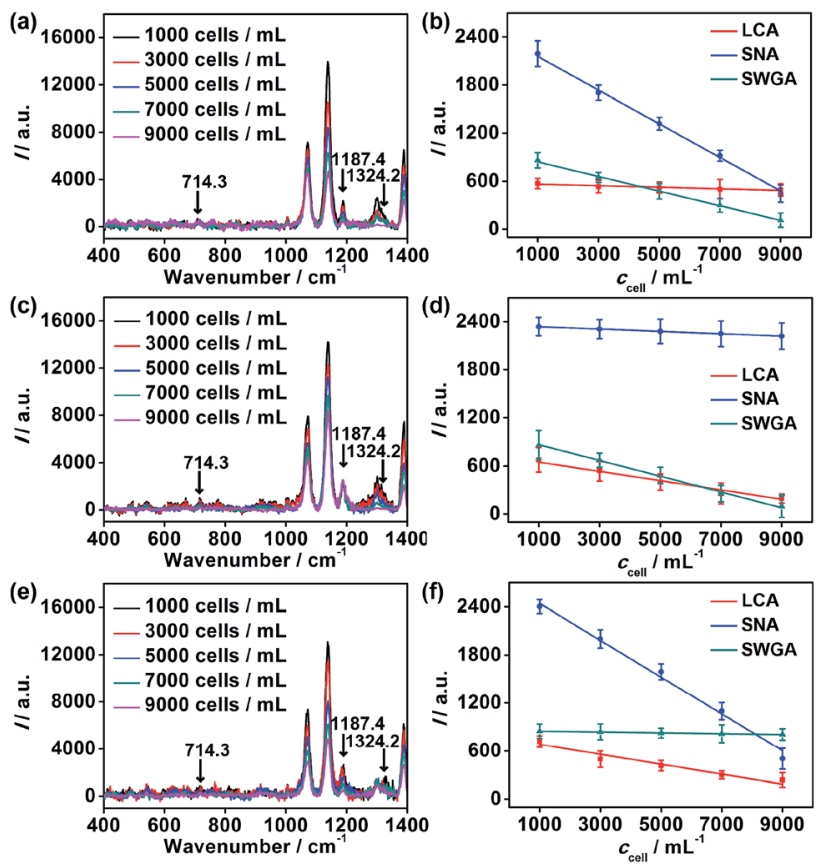

Fig. 6 Raman spectra of MGAuNS@B after competition with (a) mannosidase, (c) neuraminidase or (e) $\mathrm{N}$-acetyl-glucosaminidasetreated cells of different concentrations. Plots of Raman peak intensity at $714.3 \mathrm{~cm}^{-1}, 1187.4 \mathrm{~cm}^{-1}$ and $1324.2 \mathrm{~cm}^{-1}$ vs. (b) mannosidase, (d) neuraminidase and (f) $N$-acetyl-glucosaminidase-treated cell concentration

treated cells could be obtained (Fig. 7). Glycans cleaved by the corresponding glycan endonuclease showed an obvious decreased expression, while other two glycans varied imperceptibly. This also indicated the independence among the three pairs of recognition process in the micro-competition system. So the proposed strategy possessed the quantification capability of multiple glycans and could simultaneously monitor multiple glycan changes on living cell surfaces. By exploiting more Raman labels with well-spaced Raman bands, ${ }^{37,38}$ the multiple capability of the strategy could be expanded.

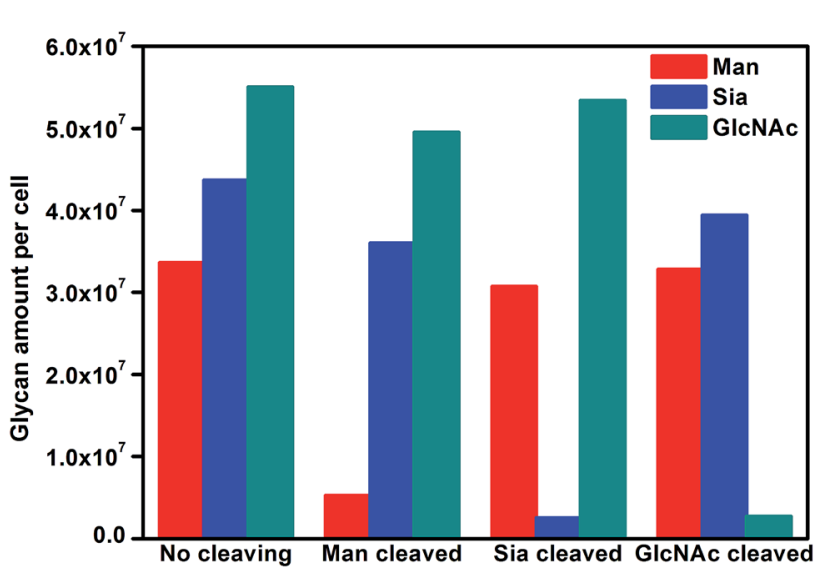

Fig. 7 Average glycan amounts on MCF-7 cells before and after cleavage treatment with Man, Sia and GlcNAc. 


\section{Conclusions}

A micro-competition system has been designed with multiplepolysaccharide-coated gold nanostars functionalized silica bubbles, target cells and Raman barcoded nanoprobes for quantification of multiple glycans on whole living cell surfaces. The nanoprobes can specifically recognize glycans on both natural cell and biomimic bubble surfaces and distinguish the corresponding Raman codes. The recognition in a homogeneous solution at a micron scale leads to enhanced competition efficiency. The AuNSs functionalized bubbles also endow the system with quick separation by buoyancy and sensitive detection by SERS. With this system a method for simultaneous Raman quantification of three types of glycans on intact cell surfaces has been developed. The regulation of multiple glycans on cell surfaces with glycan endonucleases has also been monitored in situ. The proposed strategy possesses the advantages of whole-surface accessibility, rapid detection without any cell pretreatment or labeling, convenient separation, enhanced sensitivity, high throughput and low cost. Despite the limit of available lectins and their specificity, this strategy could be expanded for other glycans or sub-types of glycans with more specific glycan-lectin interaction pairs. By combining with other biological recognition or interaction, this micro-competition system could be applied to research into the understanding of other biological interaction events.

\section{Acknowledgements}

We gratefully acknowledge National Natural Science Foundation of China (21135002, 21322506, 91213301, 91413118) and National Basic Research Program (2014CB744501). Y.C. is grateful for the support of the Scientific Research Foundation of Graduate School of Nanjing University (2013CL01) and Graduate Innovation Project of Jiangsu Province (KYZZ-0028).

\section{Notes and references}

1 I. J. Chen, H. L. Chen and M. J. Demetriou, J. Biol. Chem., 2007, 282, 35361-35372.

2 J. D. Marth and P. K. Grewal, Nat. Rev. Immunol., 2008, 8, 874-887.

3 A. Varki, Nature, 2007, 446, 1023-1029.

4 M. Pucic, S. Pinto, M. Novokmet, A. Knezevic, O. Gornik, O. Polasek, K. Vlahovicek, W. Wang, P. M. Rudd, A. F. Wright, H. Campbell, I. Rudan and G. Lauc, Glycobiology, 2010, 20, 970-975.

5 D. H. Dube and C. R. Bertozzi, Nat. Rev. Drug Discovery, 2005, 4, 477-488.

6 C. M. Szymanski, F. S. Michael, H. C. Jarrell, J. J. Li, M. Gilbert, S. Larocque, E. Vinogradov and J. R. Brisson, J. Biol. Chem., 2003, 278, 24509-24520.

7 C. Hao, L. Ding, X. J. Zhang and H. X. Ju, Anal. Chem., 2007, 79, 4442-4447.

8 M. M. Fuster and J. D. Esko, Nat. Rev. Cancer, 2005, 5, 526542.
9 K. T. Pilobello and L. K. Mahal, Curr. Opin. Chem. Biol., 2007, 11, 300-305.

10 J. F. Rakus and L. K. Mahal, Annu. Rev. Anal. Chem., 2011, 4, 367-392.

$11 \mathrm{H}$. Lis and N. Sharon, Chem. Rev., 1998, 98, 637-674.

12 M. Xie, J. Hua, Y. M. Long, Z. L. Zhang, H. Y. Xie and D. W. Pang, Biosens. Bioelectron., 2009, 24, 1311-1317.

13 W. I. Weis and K. Drickamer, Annu. Rev. Biochem., 1996, 65, 441-473.

14 P. Mitchell, Nat. Biotechnol., 2002, 20, 225-229.

15 Y. L. Chen, L. Ding and H. X. Ju, Chem. Commun., 2013, 49, 862-864.

16 Y. L. Chen, L. Ding, T. T. Liu and H. X. Ju, Anal. Chem., 2013, 85, 11153-11158.

17 G. Obaid, I. Chambrier, M. J. Cook and D. A. Russell, Angew. Chem., Int. Ed., 2012, 51, 6158-6162.

18 D. Craig, S. McAughtrie, J. Simpson, C. McCraw, K. Faulds and D. Graham, Anal. Chem., 2014, 86, 4775-4782.

19 R. Aroca, Surface-Enhanced Vibrational Spectroscopy, Wiley, Chichester, UK, 2006.

20 R. Tuma, J. Raman Spectrosc., 2005, 36, 307-319.

21 G. V. Maltzahn, A. Centrone, J. Park, R. Ramanathan, M. J. Sailor, T. A. Hatton and S. N. Bhatia, Adv. Mater., 2009, 21, 3175-3180.

22 C. L. Zavaleta, B. R. Smith, I. Walton, W. Doering, G. Davis, B. Shojaei, M. J. Natan and S. S. Gambhir, Proc. Natl. Acad. Sci. U. S. A., 2009, 106, 13511-13516.

23 R. A. Alvarez-Puebla and L. M. Liz-Marzan, Small, 2010, 6, 604-610.

24 C. G. Khoury and T. Vo-Dinh, J. Phys. Chem. C, 2008, 112, 18849-18859.

25 L. Rodriguez-Lorenzo, R. A. Alvarez-Puebla, F. J. G. Abajo and L. M. Liz-Marzan, J. Phys. Chem. C, 2010, 114, 7336-7340.

26 E. N. Esenturk and A. R. HightWalker, J. Raman Spectrosc., 2009, 40, 86-91.

27 C. Hrelescu, T. K. Sau, A. L. Rogach, F. Jäckel and J. Feldmann, Appl. Phys. Lett., 2009, 94, 153113.

28 V. L. Schmit, R. Martoglio, B. Scott, A. D. Strickland and K. T. Carron, J. Am. Chem. Soc., 2012, 134, 59-62.

29 V. L. Schmit, R. Martoglio and K. T. Carron, Anal. Chem., 2012, 84, 4233-4236.

30 J. J. Hu, R. N. Ma, F. Liu, Y. L. Chen and H. X. Ju, RSC Adv., 2014, 4, 28856-28859.

31 O. Blixt, S. Head, T. Mondala, C. Scanlan, M. E. Huflejt, R. Alvarez, M. C. Bryan, F. Fazio, D. Calarese, J. Stevens, N. Razi, D. J. Stevens, J. J. Skehel, I. Van Die, D. R. Burton, I. A. Wilson, R. Cummings, N. Bovin, C. Wong and J. C. Paulson, Proc. Natl. Acad. Sci. U. S. A., 2004, 101, 17033-17038.

32 B. T. Houseman and M. Mrksich, Chem. Biol., 2002, 9, 443454.

33 L. Ding, W. Cheng, X. J. Wang, S. J. Ding and H. X. Ju, J. Am. Chem. Soc., 2008, 130, 7224-7225.

34 L. Ding, X. R. Xiao, Y. L. Chen, R. C. Qian, L. Bao and H. X. Ju, Chem. Commun., 2011, 47, 3742-3744.

35 J. J. Lundquist and E. J. Toone, Chem. Rev., 2002, 102, 555578. 
36 P. I. Kitov and D. R. Bundle, J. Am. Chem. Soc., 2003, 125, 16271-16284.

37 Z. Y. Wang, S. F. Zong, W. Li, C. L. Wang, S. H. Xu, H. Chen and Y. P. Cui, J. Am. Chem. Soc., 2012, 134, 2993-3000.
38 C. L. Zavaleta, B. R. Smith, I. Walton, W. Doering, G. Davis, B. Shojaei, M. J. Natan and S. S. Gambhir, Proc. Natl. Acad. Sci. U. S. A., 2009, 106, 13511-13516. 